Musées, Patrimoine et Culture scientifiques et techniques

$186 \mid 2019$

novembre-décembre 2019

\title{
Les Troubles du Spectre Autistique en musée : recherches et médiation
}

Jeanne Artous

\section{OpenEdition}

\section{Journals}

Édition électronique

URL : http://journals.openedition.org/ocim/3492

DOI : 10.4000/ocim.3492

ISSN : 2108-646X

Éditeur

OCIM

Édition imprimée

Date de publication : 1 novembre 2019

Pagination : 63-64

ISSN : 0994-1908

Référence électronique

Jeanne Artous, «Les Troubles du Spectre Autistique en musée : recherches et médiation », La Lettre de I'OCIM [En ligne], 186 | 2019, mis en ligne le 01 novembre 2020, consulté le 25 janvier 2021. URL : http://journals.openedition.org/ocim/3492 ; DOI : https://doi.org/10.4000/ocim.3492

Ce document a été généré automatiquement le 25 janvier 2021.

Tous droits réservés 


\title{
Les Troubles du Spectre Autistique en musée : recherches et médiation
}

\author{
Jeanne Artous
}

Depuis la « loi handicap » du 2 février 2005, les institutions muséales se doivent de développer une politique d'accessibilité pour accueillir dans de bonnes conditions des publics aux besoins diversifiés. Nécessitant parfois de se réapproprier les codes dictés par les espaces d'exposition, l'accueil d'un public avec troubles du spectre autistique (TSA) ou en situation de handicap demande de renouveler les approches et de sortir de sa zone de confort.

2 Ma thèse questionne donc la manière dont les handicaps sensoriels - à travers l'étude des déficiences visuelles et auditives - renouvellent la conception muséographique, scénographique et notre rapport à l'art. Aussi, mon protocole de recherche est emprunté à l'ethnographie d'exposition ${ }^{1}$, à l'analyse des émotions ${ }^{2}$, à la muséologie et comprendra une étude comparative franco-canadienne du comportement des publics à travers leurs appréhensions physiques et psychiques de l'art. Ces observations de terrain questionnent la relation entre les œuvres, la scénographie et les publics et seront accompagnées d'entretiens avec les professionnels et associations. La finalité de l'étude est de développer une spatialité à la fois sociale, sensible et expérientielle promouvant l'équité entre les visiteurs.

\section{La recherche : limites et perspectives}

Outre au sein de l'environnement universitaire, je m'adresse aussi au public avec des TSA dans le cadre d'une collaboration avec l'association Les doigts qui rêvent. En partenariat avec le muséum d'Histoire naturelle de Toulouse et des professionnels issus des institutions muséales et éducatives, cette coopération permet de dégager des réflexions sur les outils de médiations culturelles pouvant être exploités par un public d'enfants aux habilités diversifiées. J'y suis chargée de la rédaction d'un document de médiation adaptée. En effet, la majorité des travaux menés sur les publics avec TSA cloisonnent les concernés à leur enfance et perdent en intensité dès lors que l'individu 
atteint l'âge adulte. Ce constat de focalisation liée à l'enfance semble pouvoir être expliqué par le fait que les observations des premiers symptômes et troubles autistiques apparaissent durant le développement infantile. Si les TSA font partie de l'individu, ils peuvent évoluer mais, de fait, on ne guérit pas de l'autisme et afin de palier un manque de références nationales et internationales, il serait intéressant et nécessaire de mener des recherches en direction du public adulte. Les recherches en neurosciences ont notamment permis d'apporter un regard nouveau sur l'autisme (Dessibourg, 2006). Elles interrogent l'hétérogénéité du trouble autistique et le fait que ce dernier varie quantitativement d'un individu à l'autre tout en pouvant être associé à d'autres affections. Les études portées par les neurosciences ont permis d'établir le croisement de données issues de la biologie, de la génétique, des interactions des aires cérébrales, ou encore du rôle des neurotransmetteurs - pour ne citer qu'eux - afin de comprendre la pluralité et la diversité des mécanismes fonctionnels, sociaux, ou comportementaux propres aux personnes avec TSA.

\section{Les réalités sur le terrain}

4 Bien que les institutions muséales soient interpellées par la diversité des publics, les offres d'accueil ne sont pas toujours présentes, ni même adaptées aux handicaps. Aussi, serait-il nécessaire de passer le cap de la sensibilisation des équipes pour se diriger vers la formation des personnels d'accueil et des médiateurs, de façon à envisager des approches et outils de médiation adaptés à la pluralité des publics et à leurs besoins. Sur le terrain, les actions de deux institutions culturelles canadiennes ont particulièrement retenu mon attention :

5 - le musée des Beaux-Arts de Montréal dont l'engagement significatif porte sur le bienêtre et l'art thérapie. L'attention portée à la typologie des publics se fait à travers le développement d'ateliers sociaux, éducatifs et communautaires proposant des réflexions autour des collections. Associé à des professionnels de santé, le musée entend être un espace inclusif : il accompagne les publics avec TSA grâce à une prise en charge spécifique, au sein d'actions adaptées ou d'ateliers préexistants. Le public avec TSA est également associé à des actions de recherches soutenues par l'institution et menées par le chercheur neurobiologiste Bruno Wicker (Wicker, 2019). S'intéressant aux mécanismes cognitifs et émotionnels, il propose une étude comparative entre public neurotypique et public avec TSA lors de leur consommation et perception de l'exposition, des œuvres et des interactions établies durant des ateliers artistiques. Mettant en évidence les divergences d'appréhension de l'art, l'étude valorise et légitimise la pluralité perceptive théorisée par Daniel Arasse (Arasse, 2000).

- le second établissement est le Centre des Sciences de Montréal qui aborde de grandes thématiques scientifiques telles que "l'humain » ou encore "l'eau dans l'univers » à travers des espaces d'expérimentation et des ateliers interactifs tinkering ( bidouillage ») où la manipulation tient une place importance. Sollicité par un groupe scolaire dont les enfants étaient porteurs de TSA, le Centre opta pour une action hors les murs et les animateurs, en collaboration avec les éducateurs, veillent à établir un contact " par étape ». La présentation des animateurs et des ateliers par exemple se fait par l'intermédiaire de la vidéo (pour ne pas imposer immédiatement une présence physique inconnue aux enfants pour qui le moindre trouble dans une journée ritualisée peut être source d'angoisses et de stress). L'animateur se déplace ensuite au sein de 
l'établissement et accompagne les enfants durant les différentes activités. Ces ateliers éducatifs expérientiels véhiculent des valeurs de partage et d'écoute où le dialogue et l'ouverture à l'autre deviennent une nécessité pour mener à bien l'exercice. Ici outil de sociabilisation, l'atelier tinkering dédramatise la perception de l'échec en l'appréhendant comme étant une étape clé à toute évolution : la résolution du problème posé n'a pas de réelle importance, ce sont l'interaction et le bon moment passé en collectivité qui importent.

7 Que ce soit avec les publics avec TSA ou en situation de handicap, l'appréhension de l'art se fait sous le prisme de leur regard, de leur sensibilité. En ce sens, il y a une réelle réciprocité dans l'échange. En interagissant avec ces publics, le regard que nous pouvons avoir sur l'art évolue : nous sommes vigilants à d'autres détails, et en conséquence, l'œuvre se construit différemment. Le partage de ces perceptions permet d'évoluer quant à la façon de réceptionner une œuvre ou une exposition.

Il y a quelques règles auxquelles il ne faut pas se soustraire. Il faut être vigilant à ne pas infantiliser la personne et en tant que médiateur, il faut pouvoir envisager le contact en sortant des codes de la communication traditionnelle. En effet, l'instauration d'un contact physique ou visuel avec une personne avec TSA peut être déstabilisante et il n'est pas toujours évident de construire une relation de bien-être ou instaurer un cadre sécuritaire. De fait, une formation auprès de spécialistes est fortement recommandée de manière à mieux comprendre ce qu'est l'autisme pour, par la suite, savoir comment réagir face à des situations qui peuvent aller à l'encontre des injonctions formulées par les institutions muséales. Ces formations, pouvant être dispensées par des associations ou des organismes spécialisés tels que le Comité national Coordination Action Handicap, permettent de faire tomber certains préjugés et nous renseignent sur l'hétérogénéité des troubles autistiques. La connaissance du handicap permet, dans le cadre professionnel, d'appréhender plus sereinement le public et d'agir calmement lors de difficultés liées à l'interaction sociale, à des problèmes communicationnels, à des troubles $\mathrm{du}$ comportement ou encore permet de savoir faire face lors de réactions sensorielles inhabituelles. Enfin, lors d'une visite de groupe organisée, il ne faut pas hésiter à prendre contact avec les éducateurs ou les accompagnateurs pour anticiper et préparer au mieux la visite et les activités pédagogiques et artistiques pouvant y être associées.

\section{NOTES}

1. Véron, E. et Levasseur, M. Ethnographie de l'Exposition, Paris : Centre Georges Pompidou, 1989.

2. Sander, D. et Varone, C. L'émotion a sa place dans toutes les expositions. La lettre de l'Ocim, $\mathrm{n}^{\circ}$ 134, 2011, pp. 22-28. 


\section{RÉSUMÉS}

En mai dernier, l'Ocim proposait des rencontres franco-québécoises à Montréal sur le thème du rôle social et inclusif des musées. Cela a permis à des professionnels québécois et français de se rencontrer et d'échanger sur l'accueil de tous types de publics en musée. À l'occasion de la publication de l'article de Cyrille Leriche sur l'accueil des publics autistes dans ce même numéro, l'Ocim a donc sollicité Jeanne Artous, doctorante à université de Lorraine et participante à ces rencontres franco-québécoises pour recueillir son point de vue sur la recherche et les actions consacrées au public présentant des troubles du spectre autistique.

INDEX

Mots-clés : Médiation, public handicapé, musée d'art

\section{AUTEUR}

\section{JEANNE ARTOUS}

Doctorante à l'université de Lorraine et participante aux rencontres franco-québécoises à Montréal sur le thème du rôle social et inclusif des musées 